\title{
Análisis geoestadístico del coeficiente de corrección por accesibilidad a núcleos de población en las valoraciones urbanísticas en Espańa
}

Rosa-María González-Ruiz. Universidad Politécnica de Madrid, Madrid, España. Sandra Martínez-Cuevas. Universidad Politécnica de Madrid, Madrid, España. Federico García-Erviti. Universidad Politécnica de Madrid, Madrid, España. María del Carmen Morillo-Balsera. Universidad Politécnica de Madrid, Madrid, España.

RESUMEN | El análisis teórico de la economía territorial se ha apoyado en la idea del territorio como espacio de intercambio, en el que la distribución de los precios del suelo rural está basada en gran medida en las rentas de accesibilidad. En este contexto se inscribe este trabajo, basado en el estudio del factor corrector por accesibilidad $\mathrm{u}_{1}$ a núcleos de población regulado en el Reglamento de valoraciones de la Ley de Suelo española. Se ha utilizado un Sistema de Información Geográfica (SIG), a partir del cual se ha desarrollado un análisis de estimación de la variable analizada mediante la aplicación de dos metodologías de interpolación, Kriging ordinario y Spline, con cuyos valores resultantes se ha podido obtener una superficie continua. Los productos del estudio han permitido un análisis de la distribución espacial del plusvalor de posición que confirma los vínculos existentes entre los desequilibrios regionales y el nivel de desarrollo económico alcanzado en cada área del territorio.

PALABRAS CLAVE | distribución espacial, geografía económica, localización.

ABSTRACT | Theoretical analysis of territorial economies has relied on the idea of territory as a space of exchange, in which the distribution of rural land prices is based largely on the income of accessibility. This paper draws from this context, and is based on the study of the correction factor for accessibility $\left(u_{1}\right)$ to regulated population centers as defined in the Valuations Regulation of the Spanish Land Law. For this, we have used Geographic Information Systems (GIS), developing two methods of spatial interpolation, Kriging and Spline, which allows the attainment of a continuous surface with the obtained values. The results of the study have allowed an analysis of the spatial distribution of added value position confirming the links between regional imbalances and the level of economic development achieved in each area of the territory.

KEYwORDs | spatial distribution, economic geography, location. 


\section{Introducción}

El análisis teórico de la economía territorial se ha apoyado en la idea del territorio como un espacio de intercambio, en el que la distribución de los precios del suelo está basada en gran medida en las rentas de accesibilidad. Las relaciones espaciales del poder político sintetizan la idea clásica de un mapa económico del territorio definido por las posibilidades de acceso a los recursos disponibles desde cada uno de los puntos que lo definen, y en el que todos los agentes compiten por el acceso a dichos recursos maximizando la utilidad de su propia posición espacial. De esta forma, cada punto del espacio presentaría un plusvalor diferencial de localización, que opera como una renta específica de posición con respecto a la situación física de los elementos que representan otros tantos atributos del valor territorial. En el contexto urbano, estos atributos, vinculados a la competencia por la ocupación del espacio como recurso limitado (Alonso, 1964), vienen determinados básicamente por la centralidad y, más concretamente, por las distancias a los centros de actividad o, históricamente, a las localizaciones de prestigio (Harvey, 1977). A su vez, el valor de las rentas de explotación estaría potenciado en el territorio rural por los costes del transporte y las distancias a los centros de producción de mano de obra o a los puntos de elaboración y distribución de materias primas. A estos elementos, Alfred Weber ańadió las economías de escala externas producidas por el efecto aglomeración (Lipietz, 1979), de modo que el factor población interviene como un atributo más del valor territorial de las rentas de explotación. Esta es la base de los modelos gravitacionales, en los que, en la determinación del plusvalor de posición de las rentas de explotación, operaría tanto la distancia a los núcleos habitados como el tamaño de la población de estos últimos (Derycke, 1971). En estos modelos socioespaciales, el valor económico de un punto en el espacio - en este caso, su plusvalor de posición- depende inversamente de la distancia a un lugar que se toma como referencia o centro gravitacional y es directamente proporcional a la magnitud de dicho centro de gravedad, cuantificada en el volumen de la población en número de habitantes (Caballer \& Roger, 2012). Esta es la base del modelo de valoración regulado en el Real Decreto 1492/2011, de 24 de octubre, por el que se aprueba el Reglamento de valoraciones de la Ley de Suelo (en adelante, RVLS), al que se refiere este trabajo. El RvLs regula la valoración urbanística del suelo, que tiene por objeto la determinación de la indemnización expropiatoria, entre otras previsiones.

Los orígenes de la relación de la localización con el valor económico del suelo han sido ampliamente estudiados por los teóricos de la economía territorial. En los siglos xviII y xix, Cantillón (1755), A. Smith (1776) y David Ricardo (1817) consideraron los efectos espaciales en la economía y Von Thünen (1783-1850) creó un modelo pionero de la localización de la actividad económica.

Posteriormente, en 1993, Walter Christaller presentó una tesis doctoral basada en un método deductivo, que se convirtió en una de las teorías geográficas con más influencia. Christaller (1966) formula la teoría de los lugares centrales, la hipótesis de un espacio isótropo, homogéneo en todas las direcciones tanto en términos de densidad demográfica como de características físicas y de infraestructura, que estructura concentraciones productivas equidistantes y áreas de mercado hexagonales. 
Por su parte, Alfred Weber (1929) postula que el principal factor de localización viene determinado por los costes de transporte, y crea el concepto de isodápana o isodapán mediante círculos concéntricos que representan el costo del transporte de un área. Finalmente, August Lösch (1954) publicó su teoría económica espacial convirtiéndose en el segundo pionero de la teoría del lugar central, después de Christaller, en cuyas teorías se inspiró.

En lo que se refiere al campo específico de la valoración agraria, diversos autores han puesto de manifiesto en España la relevancia de este fenómeno. Así, Ruiz García (1986, p. 109) introduce el concepto de las "anormalidades en las fincas que hacen precisa la corrección de su valor”, idea que a su vez extrae de Santi Juárez (1952, p. 101). Incorpora así, entre otros, los deméritos debidos a un emplazamiento geoeconómico deficiente, que, en claro precedente del RvLs, concreta en el "excesivo alejamiento de los centros urbanos" y la "falta o deficiencia de vías de comunicación”. Basa el primero de estos motivos también en Santi Juárez, quien propone una escala de depreciaciones para distintos alejamientos. Por su parte, Alonso e Iruretagoyena (1995, p. 34) se refieren a otros autores que han introducido variables de posición en la determinación del valor agrario en los métodos sintéticos, citando a Ballesteros (1991) y Salazar (1950), quienes incorporan elementos tales como las comunicaciones, las diferencias en la situación geográfica y la distancia a los centros de consumo. Los propios Alonso e Iruretagoyena ejemplifican el método sintético mediante la aplicación de un factor de corrección por distancia cuando ello supone "unos costes de transporte por desplazamiento para la realización de las labores superiores al medio”. Por su parte, Caballer (2008, p. 169) afirma que entre las características necesarias de considerar en la aplicación del método sintético de comparación espacial se encuentra "la distancia al centro urbano", factor de corrección que todos los autores incorporan como atributo en el cálculo del valor de la tierra.

La finalidad de esta investigación es realizar el estudio analítico de un parámetro, que el RVLs denomina factor de corrección por accesibilidad a núcleos de población $\left(\mathrm{u}_{1}\right)$, y que incorpora al valor del suelo rural el impacto económico de la localización, de acuerdo con las bases teóricas que antes se han citado. Actualmente, para calcular el valor del factor de corrección por accesibilidad $u_{1}$ en España se tiene que realizar un trabajo exhaustivo de creación de base de datos con la población de los municipios situados a una distancia de $4 \mathrm{~km}$ y entre 4 y $40 \mathrm{~km}$ respecto al terreno de cuyo valor se quiere calcular el factor de corrección (González Ruiz, 2012), obteniendo valores discretos de $\mathrm{u}_{1}$. Este trabajo no es sencillo, debido a que no existen repositorios con esta documentación. Para ello se ha realizado un Sistema de Información Geográfica (sIG) con datos actuales de población de todos los municipios de España, así como de las comunas y municipios de Francia y Portugal situados a una distancia máxima de $40 \mathrm{~km}$ de las fronteras respectivas. Se han georreferenciado 615 localizaciones, que corresponden a las intersecciones y los centroides de una malla de 40 x 40 que ocupa toda la España peninsular, y posteriormente se ha desarrollado un análisis geoestadístico de interpolación (Kriging) a todo el territorio. Lo relevante de nuestro estudio es que realizamos un Kriging que permite un cálculo y corrección superior de los valores $\mathrm{u}_{1}$, pues interpola y calcula este factor en cualquier punto de la geografía 
española teniendo en cuenta los valores discretos de $\mathrm{u}_{1}$. Es decir, pasamos de unos valores discretos de $\mathrm{u}_{1}$ a una superficie continua que contiene todos los valores de dicho factor. Al mismo tiempo, el Kriging permite ver el mapa espacial continuo por zonas o categorías de los valores del factor $\mathrm{u}_{1}$, detectando de forma visual dónde se encuentran, por ejemplo, los valores extremos del factor $\mathrm{u}_{1}$, bien por provincias o municipios o en otras situaciones del estudio, sin necesidad de realizar cálculos complejos con las fórmulas marcadas por la legislación española.

Con los resultados obtenidos se pretende conocer el valor de este factor de corrección por accesibilidad en todo el territorio espańol, sirviendo de apoyo al legislador, que puede obtener conclusiones que afecten a la ordenación territorial y urbanística del país. Asimismo, los resultados de este trabajo suponen una contribución al análisis territorial del impacto económico del valor de posición en las rentas de explotación en el suelo rural. Además, constituyen un avance en los procesos de elaboración de las hojas de aprecio en los procedimientos expropiatorios de suelo rural, tanto por los peritos de los propietarios como por los de las administraciones públicas expropiantes.

\section{Datos y método}

El RvLs dispone que, en el suelo rural, los terrenos se valoran mediante la capitalización de la renta anual real o potencial de la explotación agropecuaria, forestal o extractiva que se desarrolle en dicho suelo (art. 7.1). Además, esta norma ańade que la valoración final del suelo por este procedimiento deberá tener en cuenta la localización espacial concreta del inmueble, y aplicar, cuando corresponda, un factor global de corrección al valor de capitalización (art. 17.1). Este factor, destinado a ponderar la renta de posición del precio de la tierra en el medio rural, se obtiene según la siguiente fórmula:

donde:

$$
V f=V \times F l
$$

$\mathrm{Vf}=$ Valor final del suelo, en euros.

$\mathrm{V}=$ Valor de capitalización de la renta de la explotación, en euros.

$\mathrm{Fl}=$ Factor global de localización.

El factor global de localización Fl, de acuerdo con el art. 17.2 RVLS, deberá obtenerse del producto de los tres factores de corrección que se mencionan a continuación y no podrá ser superior a dos:

a. Por accesibilidad a núcleos de población, $\mathrm{u}_{1}$.

b. Por accesibilidad a centros de actividad económica, $u_{2}$.

c. Por ubicación en entornos de singular valor ambiental o paisajístico, $\mathrm{u}_{3}$.

De forma que:

$$
\mathrm{F} 1=\mathrm{u}_{1} \times \mathrm{u}_{2} \times \mathrm{u}_{3}
$$


La Sentencia 141/2014, de 11 de septiembre de 2014, del Tribunal Constitucional, declaró la inconstitucionalidad y, por tanto, la nulidad del inciso "hasta un máximo del doble" del Texto refundido de la Ley de suelo, aprobado por Real Decreto Legislativo 2/2008 (posteriormente sustituido por el Real Decreto Legislativo 7/2015), precedente del inciso "no podrá ser superior a dos" del art. 17.2 RVLS. Por tanto, debe entenderse que también queda sobrevenida la nulidad de dicho inciso, ya que el RvLs desarrolla el Texto refundido.

Entre los factores que intervienen en el factor global de localización se encuentra el factor de corrección por accesibilidad a núcleos de población, definido en el art. 17.3 RVLS por la siguiente fórmula:

donde:

$$
\mathbf{u}_{1}=1+\left[\mathrm{P}_{1}+\frac{\mathrm{P}_{2}}{3}\right] \times \frac{1}{1.000 .000}
$$

$\mathrm{P}_{1}$ es el número de habitantes a una distancia de $4 \mathrm{~km}$ del suelo a valorar.

$\mathrm{P}_{2}$ es el número de habitantes a una distancia entre 4 y $40 \mathrm{~km}$ del suelo a valorar.

El flujo de trabajo desarrollado en esta investigación para la consecución de los objetivos es el siguiente: inicialmente se delimita el área de estudio y se crea una base de datos (población y municipios) que serán representados posteriormente en un sig. Seguidamente se realiza el cálculo de los valores $\mathrm{P}_{1}, \mathrm{P}_{2}$ y $\mathrm{u}_{1}$, así como su geolocalización, para crear un modelo de superficie usando métodos geoestadísticos.

\section{Área de estudio}

El ámbito de estudio de donde se han obtenido los datos es el territorio peninsular de España, que abarca una extensión de $492.197 \mathrm{~km}^{2}$, y la población total de 43.731.572 habitantes. En la figura 1a) puede verse la extensión del territorio y la ubicación de Espańa al suroeste de Europa, colindando con Francia al norte y Portugal al oeste.

Previo a la creación de la base de datos, se descargó toda la información necesaria disponible en distintos organismos oficiales: centro de descargas del Centro Nacional de Información Geográfica (CNIG) del Instituto Geográfico Nacional (IGN) del Ministerio de Fomento; sig Nacional de España, del IGN del Ministerio de Fomento (signa); catálogo de metadatos de la Infraestructura de Datos Espaciales (IDE) de España, del Ministerio de Fomento; y finalmente, del Banco de Datos de la Naturaleza (BDN) del Ministerio de Agricultura, Alimentación y Medio Ambiente. Seguidamente se realizó el cálculo de los valores $\mathrm{P}_{1}, \mathrm{P}_{2}$ y $\mathrm{u}_{1}$ y su geolocalización, para crear un modelo de superficie usando métodos geoestadísticos. Estas técnicas geoestadísticas han sido utilizadas en diferentes estudios de suelo. Weih y Dick (2008) han aplicado en este caso métodos de interpolación para calcular la probabilidad de las diferentes especies de árboles que se encuentran en el paisaje.

Las dimensiones de la malla de 40 x $40 \mathrm{~km}$ que ocupa todo el territorio peninsular espańol se deben a que, en la fórmula de cálculo recogida en la legislación, la máxima distancia de cálculo se sitúa a $40 \mathrm{~km}$ del punto en el que se realiza la valoración. Se calcula la población situada a $4 \mathrm{~km}$ y $40 \mathrm{~km}$ del punto valorado, resultando un total de 615 puntos. El cálculo se ha realizado para cada coordenada X, Y, tanto de la intersección de la malla de 40 x $40 \mathrm{~km}$ como de los centroides de la misma (figura 1b). 
FIgURA I | (a) Localización de España en Europa

(b) Distribución de la malla en la península y de los centroides de los puntos
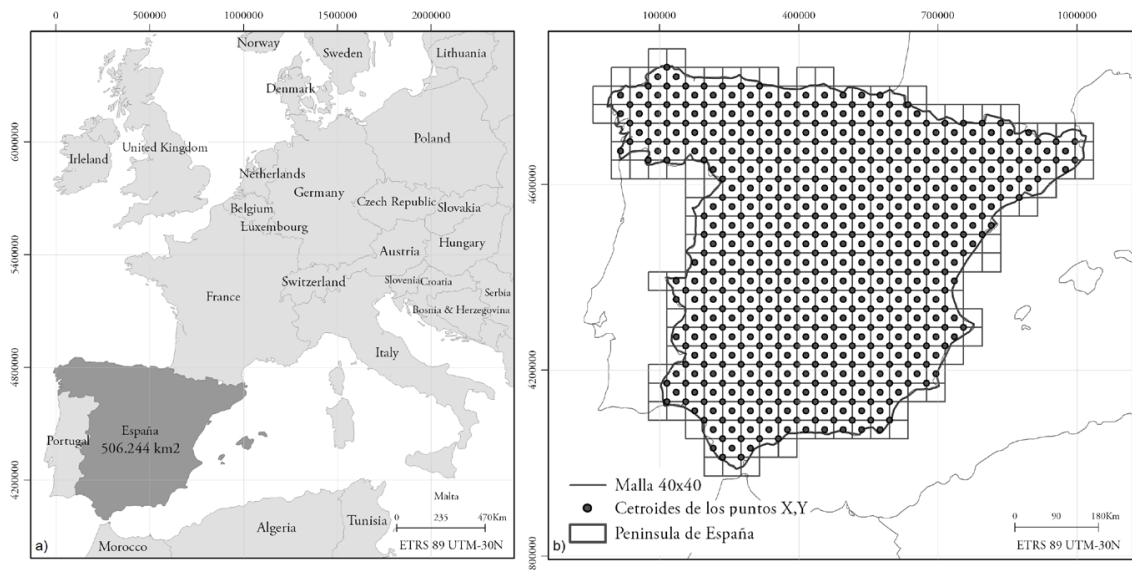

FUENTE ELABORACIÓN PROPIA

\section{Base de datos}

Debido a la importancia en el desarrollo de las bases de datos aplicadas en un Sistema de Información Geográfico (Goodchild \& Haining, 2004), para la aplicación de procedimientos de análisis espacial se ha construido una base de datos con la información anteriormente mencionada, que se considera la más adecuada para el propósito perseguido. En la tabla 1 puede verse el nombre abreviado de los atributos, así como su descripción, tipo y categorización.

TABLA I | Base de datos con los atributos objeto de estudio

\begin{tabular}{|c|c|c|c|c|}
\hline $\begin{array}{c}\text { NOMBRE } \\
\text { ABREVIADO }\end{array}$ & GEOMETRÍA & ETIQUETA & DESCRIPCIÓN & TIPO \\
\hline X & Punto & Coordenada X & $\begin{array}{l}\text { Localización geográfica. Coordena- } \\
\text { da horizontal. El sistema utilizado } \\
\text { es: Sistema de proyección (UTM) }\end{array}$ & Numérica \\
\hline Y & Punto & Coordenada Y & $\begin{array}{l}\text { Localización geográfica. } \\
\text { Coordenada vertical. El sistema } \\
\text { utilizado es: (UTM) }\end{array}$ & Numérica \\
\hline$P_{1}$ & Punto & $\begin{array}{l}\text { Cantidad de población } \\
\text { a } 4 \mathrm{~km} \text { de cada punto }\end{array}$ & $\begin{array}{l}\text { Cantidad de población a } 4 \mathrm{~km} \text { de } \\
\text { cada punto }\end{array}$ & Numérica \\
\hline$P_{2}$ & Punto & $\begin{array}{l}\text { Cantidad de población } \\
\text { a } 40 \mathrm{~km} \text { de cada punto }\end{array}$ & $\begin{array}{l}\text { Cantidad de población a } 40 \mathrm{~km} \text { de } \\
\text { cada punto }\end{array}$ & Numérica \\
\hline $\mathrm{u}_{1}$ & Punto & $\begin{array}{l}\text { factor de corrección por } \\
\text { accesibilidad }\end{array}$ & $\begin{array}{l}\text { Valor del factor de corrección por } \\
\text { accesibilidad }\end{array}$ & Numérica \\
\hline $\mathrm{Mu}$ & Polígono & Municipios & $\begin{array}{l}\text { Delimitación de los municipios de } \\
\text { Espańa }\end{array}$ & Categórica \\
\hline CCAA & Polígono & $\begin{array}{l}\text { Comunidades Autó- } \\
\text { nomas }\end{array}$ & $\begin{array}{l}\text { Delimitación de las Comunidades } \\
\text { Autónomas }\end{array}$ & Categórica \\
\hline $\operatorname{Pr}$ & Polígono & Provincias & Provincias de España & Categórica \\
\hline
\end{tabular}


(continuación)

\begin{tabular}{|l|l|l|l|l|}
\hline $\begin{array}{c}\text { NOMBRE } \\
\text { ABREVIADo }\end{array}$ & GEOMETRÍA & \multicolumn{1}{|c|}{ ETIQUETA } & \multicolumn{1}{c|}{ DESCRIPCIÓN } & \multicolumn{1}{c|}{ TIPO } \\
\hline PL & Polígono & Países Limítrofes & $\begin{array}{l}\text { Países colindantes Portugal, Francia } \\
\text { y Marruecos }\end{array}$ & Categórica \\
\hline PobNu & Polígono & Cantidad de Población & Población Núcleos & Numérica \\
\hline PobPL & Polígono & Cantidad de Población & $\begin{array}{l}\text { Población de los países limítrofes, } \\
\text { Francia y Portugal }\end{array}$ & Numérica \\
\hline
\end{tabular}

FUENTE ELABORACIÓN PROPIA

Con esta base de datos espacial se ha construido una plataforma que facilitará el análisis espacial y nos permitirá visualizar los datos en los mapas que se detallan en la siguiente sección.

Análisis espacial del factor de corrección por accesibilidad a núcleos de población $\mathbf{u}_{1}$ Todos los puntos fueron almacenados y representados en un Sistema de Información Geográfica. En cada uno de los puntos se han calculado los valores $\mathrm{P}_{1}$ y $\mathrm{P}_{2}$, el número de habitantes a una distancia de $4 \mathrm{~km}$ y el número de habitantes situados a más de 4 $\mathrm{km}$ y a menos de $40 \mathrm{~km}$. Puesto que en la fórmula del cálculo del factor de corrección por accesibilidad a núcleos de población $\mathrm{u}_{1}$ los valores que se utilizan son los de $\mathrm{P}_{1}$ y los valores de $\mathrm{P}_{2}$ divido por 3, para comparar el peso que tienen en la ecuación [2] ambos valores, se ha representado el valor de $\mathrm{P}_{1}$ y $\mathrm{P}_{2} / 3$. Además, se realizó un análisis de categorización de los valores de $\mathrm{P}_{1}$ y $\mathrm{P}_{2} / 3$, con el objetivo de obtener una variabilidad representativa de los datos en todas las Comunidades Autónomas.

Se pudo apreciar cómo el peso del valor $\mathrm{P}_{2} / 3$ es mucho más elevado. Los mayores valores en ambos casos se dan en las capitales de provincias. En $\mathrm{P}_{2} / 3$ los valores superiores se encuentran en toda la costa espańola, aumentando en la zona de Levante y Cataluña. También habría que destacar el valor en la Comunidad de Madrid y el eje que se crea con el valor elevado del coeficiente $\mathrm{P}_{2} / 3$ uniendo las ciudades de Santander y Castellón de la Plana, pasando por Zaragoza.

Con los datos resultantes del total de habitantes situados a la distancia de 4 $\mathrm{km}\left(\mathrm{P}_{1}\right)$ y el total de habitantes situados entre $4 \mathrm{~km}$ y $40 \mathrm{~km}\left(\mathrm{P}_{2}\right)$, se ha procedido al cálculo del factor de corrección por accesibilidad a núcleos de población $\mathrm{u}_{1}$, mediante la fórmula del art. 17.2 del RvLs.

Los datos obtenidos para el valor de $\mathrm{u}_{1}$ de cada uno de los puntos se han representado en la figura 2. En este caso se utilizó la categorización de progresión geométrica, ya que los datos crecen muy lentamente en los valores bajos y de forma brusca en los altos.

En cuanto a la distribución territorial del valor $\mathrm{u}_{1}$, ha podido comprobarse que en la Comunidad Autónoma de Madrid se encuentra el mayor valor de $\mathrm{u}_{1}$, y en ella también se localiza un mayor número de puntos con dicho valor. Por otro lado, la Comunidad Autónoma de Cataluńa tiene una serie de focos con un valor de $\mathrm{u}_{1}$ muy elevado, aunque en gran parte de su extensión el valor del factor se encuentra en un rango muy bajo. Tanto la Comunidad Valenciana como la Comunidad Autónoma Vasca resultan bastante homogéneas en sus resultados, encontrándose en un valor medio. La Comunidad Autónoma de Andalucía presenta unos valores más elevados 
en Sevilla, Cádiz y Málaga, y a partir de estos valores el resto es muy homogéneo y decreciente; teniendo en cuenta también los valores obtenidos en Córdoba, Almería y Huelva, en general se trata de valores homogéneos. Las Comunidades Autónomas de Aragón, Cantabria, Principado de Asturias, Galicia, Castilla y León y la Comunidad Foral de Navarra presentan unos valores de $\mathrm{u}_{1}$ ligeramente superiores en las capitales de las respectivas provincias y el resto del territorio tiene unos valores bajos. La Comunidad Autónoma de Castilla La Mancha tiene unos valores de $\mathrm{u}_{1}$ reducidos; solamente en las zonas próximas a las Comunidades Autónomas de Madrid y Valenciana los suelos elevan su valor por la influencia de la población de estas últimas.

\section{FIGURA 2 Distribución del factor de corrección por accesibilidad $\mathbf{u}_{1}$}

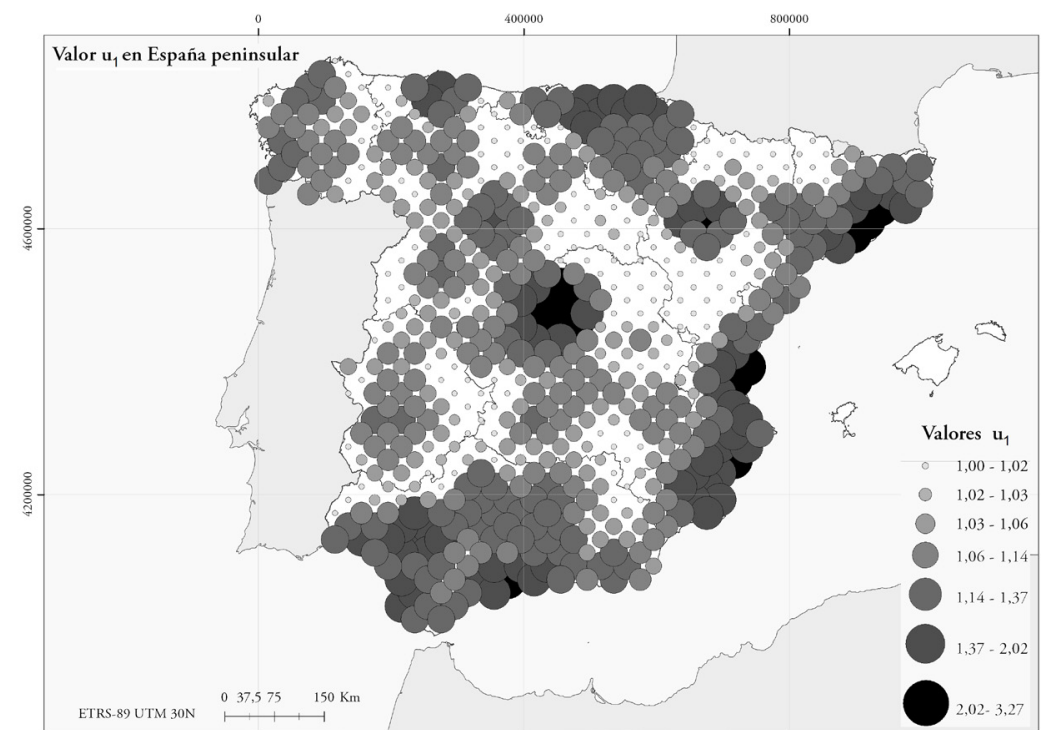

FUENTE ELABORACIÓN PROPIA

Para obtener una homogeneización de la zona según el factor de corrección por accesibilidad $\mathrm{u}_{1}$, se han estudiado dos interpolaciones de tipo local, definiendo las características diferenciadoras de las zonas y empleando para ello la estadística multivariante. La realización de la categorización se llevó a cabo utilizando el programa ArcGis_v10.1.

\section{Estadística espacial}

Entendemos por dato espacial todo aquel que tiene asociada una referencia geográfica, de tal modo que podemos localizar exactamente dónde sucede dentro de un mapa (Haining, 2003). En este trabajo, se utiliza dos interpolaciones de tipo local: la primera basada en medias ponderadas, el Kriging, método desarrollado en el marco de la teoría geoestadística; y el método Spline, el cual ajusta funciones polinómicas en las que las variables independientes son X e Y. 
La geoestadística es una rama de la estadística aplicada que se especializa en el análisis y la modelación de la variabilidad espacial en ciencias de la tierra. Los métodos empleados en este trabajo para la estimación del factor de corrección por accesibilidad $\mathrm{u}_{1}$ corresponden al análisis geoestadístico (Díaz-Viera, 2002).

La información almacenada en la base de datos espacial ha sido fundamental para la categorización espacial del factor de corrección por accesibilidadn $\mathrm{u}_{1}$, partiendo de los estadísticos preliminares de primer orden como la media, rango, desviación estándar, etcétera. Los valores que adquieren estos parámetros en el presente estudio se exponen en la sección de aplicación. A continuación, se realizó una modelización de la variabilidad espacial del factor de corrección por accesibilidad $\mathrm{u}_{1}$, lo que se conoce como semivariograma experimental, el cual es obtenido por estimación a partir de los datos de una muestra y nos indica la variación de la correlación entre los datos en función de la distancia. El estimador más común se basa en el método de los momentos, y también puede escribirse mediante la expresión siguiente (Cressie, 1990):

$$
\gamma(h)=\frac{1}{2|N(h)|_{N(h)}} \Sigma\left(Z\left(x_{i}\right)-Z\left(x_{j}\right)\right)^{2}, h \in R^{d}
$$

donde:

$Z\left(x_{i}\right)=$ valor de la variable en el punto $x_{i}$

$Z\left(x_{i}\right)=$ valor de la variable en el punto $x_{j}$

$N(h)=\left\{\left(\mathrm{x}_{\mathrm{i}}, \mathrm{x}_{\mathrm{j}}\right) / \mathrm{x}_{\mathrm{i}}-\mathrm{x}_{\mathrm{j}}=\mathrm{h} ; \mathrm{i}, \mathrm{j}=1, \ldots, \mathrm{n}\right\}$

$N\left(h_{\theta}\right)=$ número de pares distintos, separados por una distancia h en la dirección $\theta$.

Para calcular el semivariograma experimental omnidireccional (semivariograma válido para todas las direcciones) se realizan pruebas con diversos valores de la distancia h (lag), y con distintas tolerancias sobre los mismos, hasta conseguir obtener un semivariograma omnidireccional adecuado. En caso de no encontrar un variograma omnidireccional óptimo, se debe emplear otra medida distinta de la continuidad espacial. Conseguido el semivariograma omnidireccional, deben encontrarse los posibles patrones de anisotropía, calculando los semivariogramas direccionales. Así pues, el semivariograma experimental se obtiene de la regionalización observada. Estos semivariogramas pueden no satisfacer los requisitos para tener dicha categoría, por lo que deben ser ajustados a uno de los modelos teóricos que los cumplen. Las características básicas del semivariograma son:

- Alcance: distancia para la cual se estabiliza el variograma. Representa la zona de influencia de un dato y es la distancia a partir de la cual no hay correlación.

- Meseta: valor constante que toma el semivariograma a distancias mayores al alcance.

- Efecto Pepita: valor del semivariograma en el origen.

Los modelos teóricos de variogramas son curvas, generadas a partir de una función matemática, que ajustaremos a nuestros datos y nos permitirán conocer la distribución para todos los puntos en el espacio. Los modelos básicos más usados se 
desarrollan construyendo matemáticamente una función aleatoria y calculando su semivariograma teóricamente, imponiéndose la condición de función condicionada positiva (Moral-García, 2003). Estos son:

- Esférico:

$$
\gamma(h)=\left\{\begin{array}{l}
\frac{m}{2}\left[3\left(\frac{h}{a}\right)-\left(\frac{h}{a}\right)^{3}\right] \text { si } 0 \leq h<a \\
m \text { si } h=a
\end{array}\right.
$$

siendo $\mathrm{m}$ y a, constantes positivas, el valor de la meseta (m) y del alcance (a), respectivamente.

- Exponencial:

$$
\gamma(h)=m\left(1-e^{-\frac{h}{a}}\right) \text { si } h>0
$$

siendo $\mathrm{m}$ y a, constantes positivas, el valor de la meseta (m) y del alcance (a), respectivamente.

- Gaussiano:

$$
\gamma(h)=m\left(1-e^{-\frac{h^{2}}{a^{2}}}\right) \text { si } h \geq 0
$$

siendo h y a, constantes positivas, el valor de la meseta (m) y del alcance (a), respectivamente.

Posteriormente, se ha llevado a cabo un análisis de estimación de la variable factor de corrección por accesibilidad $\mathrm{u}_{1}$, mediante el estudio de dos tipos de interpolación espacial (Kriging y Spline con tensión), con el fin de conseguir una superficie continua con las estimaciones, lo que permite conocer valores de la variable indicada allí donde no fue muestreada. La interpolación de datos ofrece la ventaja de proyectar mapas o superficies continuas a partir de datos discretos (Johnston, Ver Hoef, Krivoruchko \& Lucas, 2001). La precisión en el mapa generado, a partir de las características por estudiar, depende en gran medida de la estructura espacial de los datos. Se estima que donde más fuerte es la correlación espacial de los valores de la variable en estudio, mejor es la calidad del mapeo (Kravchenko, 2003). Algunos estudios, como Pebesma (2006), introducen variables externas (tamaño de forma, configuración de la forma) en estudios geoestadísticos para predicción espacial.

\section{Kriging}

Una completa introducción a Kriging fue dada por Cressie (1990). El problema de la estimación de los atributos en los lugares no muestreados se favorece de forma especial cuando se considera la existencia de un modelo de dependencia espacial. Las variables naturales se distribuyen en el espacio de una forma continua, ley de Tobler, que dice: “Todas las cosas están relacionadas entre sí, pero las cosas más próximas en el espacio tienen una relación mayor que las distantes” (Tobler, 1970), lo cual suele cumplirse en la naturaleza. 
El Kriging es el nombre genérico utilizado por los usuarios de la geoestadística para denominar a una familia de algoritmos de regresión mediante mínimos cuadrados, en reconocimiento al trabajo pionero de Daniel Krige, en 1951.

Todos los estimadores del tipo krigeaje no son sino variantes del estimador lineal básico, definido como:

$$
Z^{*}(x)-m(x)=\sum_{i=1}^{n} \omega_{i}\left[Z\left(x_{i}\right)-m\left(x_{i}\right)\right]
$$

donde,

$\omega_{i}$ son los pesos asignados a los datos $z\left(x_{i}\right)$, siendo estos realizaciones de la variable aleatoria $Z\left(x_{i}\right)$.

Los valores esperados de las variables aleatorias $Z(x)$ y $Z\left(x_{i}\right)$ son $m(x)$ y $m\left(x_{i}\right)$, respectivamente.

Debido a que tanto los valores desconocidos $z(x)$, como los datos $z\left(x_{i}\right)$, son realizaciones de las variables aleatorias $Z(x)$ y $Z\left(x_{i}\right)$, se puede definir el error de la estimación $Z^{*}(x)-Z(x)$ como variable aleatoria.

Todos los tipos de Kriging comparten el objetivo de minimizar la varianza del error (o de estimación) $\sigma_{E}^{2}(x)$, con la restricción de ser un estimador insesgado, o sea:

$$
\sigma_{E}^{2}(x)=\operatorname{Var}\left[Z^{*}(x)-Z(x)\right] \rightarrow \text { Minimo }
$$

con la restricción,

$$
E=\left[Z^{*}(x)-Z(x)\right]=0
$$

Las clases de Kriging varían en función del modelo adoptado para la función aleatoria $Z(x) Z(x)$ (Moral García, 2003).

El Kriging ordinario es la interpolación recomendada para patrones de puntos de una región, con el fin de obtener resultados más razonables (Ghiasi \& Nafisi, 2016).

El Kriging ordinario asume que la variable es estacionaria de segundo orden, con media $\mu$ constante, aunque desconocida, función de varianza $\mathrm{C}(\mathrm{h})$ conocida o una función aleatoria intrínsecamente estacionaria, también con media $\mu$ constante y desconocida, pero cuya varianza no está acotada (Montero \& Larraz, 2008).

$$
\mathrm{Z}^{*}(\mathrm{x})=\sum_{\mathrm{i}=1}^{\mathrm{n}} \omega \mathrm{Z}(\mathrm{s})
$$

Los $n$ pesos $\omega_{i}$ deben determinarse minimizando la varianza del error, con la restricción de ser una estimación insesgada (Moral-García, 2003).

El Kriging es un método de estimación óptimo desde el punto de vista estadístico, siempre que se cumplan algunas condiciones. La más importante es que el semivariograma tenga validez general para el modelo; es decir, que la interdependencia entre los datos debe ser función exclusivamente de la distancia entre ellos (de su posición relativa) y no de su localización espacial absoluta.

La validación cruzada utiliza todos los datos muestrales para estimar el modelo de autocorrelación. Cada vez quita un dato muestral y lo predice con el resto de la muestra, después compara el valor predicho de ese punto con el valor observado. Este 
procedimiento se repite con cada uno de los puntos muestrales y al final la validación cruzada compara los valores observados con los calculados (Moreno Jiménez, 2007).

\section{Spline}

La técnica de Spline consiste en el ajuste local de ecuaciones polinómicas en las que las variables independientes son X e Y. La forma de la superficie final va a depender de un parámetro de tensión que hace que el comportamiento de la superficie interpolada tienda a asemejarse a una membrana más o menos tensa o aflojada que pasa por los puntos de observación.

El algoritmo utilizado para la interpolación de la superficie se basa en la siguiente fórmula:

donde:

$$
\mathrm{S}(\mathrm{x}, \mathrm{y})=\mathrm{T}(\mathrm{x}, \mathrm{y})+\sum_{\mathrm{j}=1}^{\mathrm{N}} \lambda \mathrm{R}(\mathrm{r})
$$

$\mathrm{j}=1,2, \ldots, \mathrm{N}$

$\mathrm{N}$ es la cantidad de puntos.

$\lambda \mathrm{j}$ son coeficientes determinados por la solución de un sistema de ecuaciones lineales. rj es la distancia del punto $(x, y)$ al punto $j$.

$\mathrm{T}(\mathrm{x}, \mathrm{y})$ y $\mathrm{R}(\mathrm{r})$ se definen para la opción utilizada.

La interpolación Spline se produce con tensión, de forma que los cambios bruscos de valores pueden presentar oscilaciones artificiales significativas (Mitášová \& Hofierka, 1993). La incorporación de la tensión en una dirección permite añadir anisotropía al proceso de interpolación (Schweikert, 1966). Por tanto:

donde:

$$
\mathrm{T}(\mathrm{x}, \mathrm{y})=\mathrm{a} 1
$$

a1 es un coeficiente determinado por la solución de un sistema de ecuaciones lineales.

$$
\mathrm{R}(\mathrm{r})=-\frac{1}{2 \pi \varphi^{2}} \ln \frac{\mathrm{r} \varphi}{2}+\mathrm{c}+\mathrm{K}(\mathrm{r} \varphi)
$$

donde:

$r$ es la distancia entre el punto y la muestra.

$\varphi 2$ es el parámetro de peso.

Ko es la función de Bessel modificada.

c es una constante igual a 0,577215 .

\section{Aplicación de los modelos al territorio peninsular espańol}

Para la estimación del factor de corrección por accesibilidad $\mathrm{u}_{1}$, se aplicaron dos interpolaciones a la zona de estudio: el método del Kriging ordinario y la técnica de Spline. Los datos estadísticos de partida de la muestra de estudio, respecto a la variable factor de corrección por accesibilidad $\mathrm{u}_{1}$, son los siguientes: Muestra: 615; Mínimo: 1,01; 
Máximo: 3,27; Media: 1,14; Rango: 2,13; Quartil Q1: 1,00; Mediana Q2: 1,06; Quartil Q3: 1,15; Desviación estándar: 0,25; y Coeficiente Variación: 21,92\%

Con los valores obtenidos se puede concluir que los valores del factor $\mathrm{u}_{1}$ no siguen una distribución normal y que presentan una simetría positiva respecto a la media, y que la cola de la distribución se alarga para valores superiores a la media. Además, el $75 \%$ de los valores del factor $\mathrm{u}_{1}$ tiene valores de 1,15 o menores. Los valores máximos corresponden a las zonas de la Comunidad de Madrid y de Barcelona, y sus respectivas áreas metropolitanas.

A continuación se expone una figura con una tabla de descriptivos del factor de corrección por accesibilidad $\left(\mathrm{u}_{1}\right)$ donde se recogen los valores obtenidos en cada una de las Comunidades Autónomas. Y dos gráficos de la variable factor de corrección por accesibilidad: figura 3a) Diagrama de frecuencias de los valores $\mathrm{u}_{1}$, donde se puede observar que $75 \%$ de los valores son menores 1,15; y figura $3 \mathrm{~b}$ ) Diagrama de Box-plot., donde hay 61 valores extremos, y que representa 9,9\% de los datos. La mediana tiene un valor de 1,06. Los extremos de la caja representan el Q1 y Q3.

FIGURA 3 Tabla de descriptivos del factor de corrección por accesibilidad ( $\mathbf{u}_{1}$ ) en las Comunidades Autónomas. (a) Diagrama de frecuencias de los valores $\mathbf{u}_{1}$. (b) Diagrama de Box-Plot

\begin{tabular}{|l|c|c|c|c|c|c|c|c|c|c|}
\hline Comunidades Aut. & Muestras & Mínimo & Máximo & Rango & Media & DesTip & Q1 & Q2 & Q3 & Coef.Var (\%) \\
\hline Andalucía & 113 & 1.01 & 1.96 & 0.96 & 1.17 & 0.17 & 1.05 & 1.12 & 1.22 & 14.53 \\
\hline Aragón & 58 & 1.01 & 1.92 & 0.92 & 1.07 & 0.14 & 1.01 & 1.02 & 1.04 & 13,08 \\
\hline Asturias & 12 & 1.01 & 1.31 & 0.30 & 1.12 & 0.12 & 1.02 & 1.09 & 1.23 & 10.7 \\
\hline Cantabria & 7 & 1.02 & 1.17 & 0.16 & 1.09 & 0.07 & 1.02 & 1.06 & 1.17 & 6.42 \\
\hline Castilla la Mancha & 99 & 1 & 2.27 & 1.27 & 1.07 & 0.15 & 1.02 & 1.04 & 1.08 & 14.02 \\
\hline Castilla León & 116 & 1.01 & 1.49 & 0.48 & 1.06 & 0.07 & 1.02 & 1.04 & 1.08 & 6.60 \\
\hline Cataluña & 39 & 1.01 & 2.93 & 1.92 & 1.26 & 0.43 & 1.05 & 1.13 & 1.23 & 34.12 \\
\hline Extremadura & 52 & 1.01 & 1.14 & 0.14 & 1.04 & 0.03 & 1.02 & 1.04 & 1.06 & 2.88 \\
\hline Galicia & 36 & 1.02 & 1.19 & 0.18 & 1.08 & 0.05 & 1.03 & 1.07 & 1.09 & 4.63 \\
\hline Madrid & 11 & 1.02 & 3.25 & 2.23 & 2.09 & 0.84 & 1.37 & 2.01 & 3.02 & 40.19 \\
\hline Murcia & 13 & 1.04 & 1.69 & 0.66 & 1.26 & 0.23 & 1.08 & 1.10 & 1.48 & 18.25 \\
\hline Navarra & 14 & 1.02 & 1.35 & 0.33 & 1.23 & 0.08 & 1.07 & 1.23 & 1.15 & 6.50 \\
\hline País Vasco & 8 & 1.19 & 1.52 & 0.33 & 1.34 & 0.14 & 1.22 & 1.32 & 1.50 & 10.45 \\
\hline Rioja & 6 & 1.02 & 1.13 & 0.11 & 1.08 & 0.04 & 1.04 & 1.10 & 1.12 & 3.70 \\
\hline Valencia & 31 & 1.02 & 1.91 & 0.89 & 1.33 & 0.30 & 1.06 & 1.22 & 1.69 & 22,56 \\
\hline
\end{tabular}
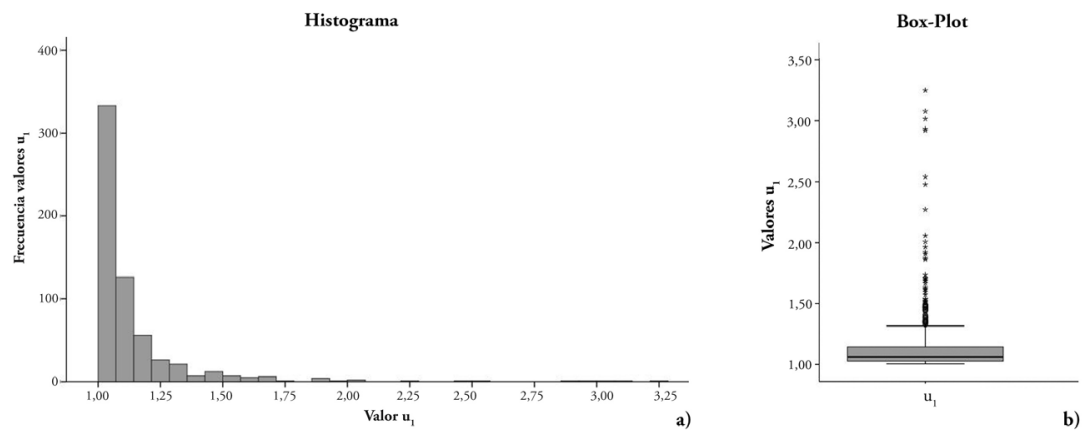

FUENTE ELABORACIÓN PROPIA 
Dentro del estudio exploratorio de los datos se busca si hay tendencias globales del factor en estudio. Para ello se realizó un análisis gráfico de tendencia global del factor $\mathrm{u}_{1}$ sobre los planos este-oeste $(\mathrm{X}-\mathrm{Z})$ y otro norte-sur $(\mathrm{Y}-\mathrm{Z})$, concluyendo que hay una débil tendencia global en la dirección este-oeste, que no fue considerada en el estudio.

\section{Kriging ordinario}

A continuación se realizó el análisis estructural de los datos mediante la interpolación del Kriging ordinario. Para encontrar el mejor variograma experimental que definiera la continuidad espacial de los valores de la variable factor de corrección por accesibilidad $\mathrm{u}_{1}$, se realizaron diferentes pruebas con diferentes tamańos del lag, así como del número de lags, adoptándose a diferentes azimut para estudiar una posible anisotropía. De los resultados del estudio pudo comprobarse que no había anisotropía, ya que a partir de distintos azimut no se observan cambios significativos en el variograma o semivariograma teórico.

En la tabla 2a) pueden verse las características del variograma experimenta y en la tabla 2b) la eficiencia del modelo (validación cruzada del modelo Kriging ordinario).

TABLA 2 (a) Variograma experimental. (b) Validación cruzada

\begin{tabular}{|l|l|}
\hline \multicolumn{1}{|c|}{ DATOS } & EXPONENCIAL \\
\hline Vecinos máximos & 5 \\
\hline Vecinos mínimos & 2 \\
\hline Sector & 4 \\
\hline Anisotropía & No \\
\hline Alcance o rango & $201.635 \mathrm{~m}$ \\
\hline Nugget & 0 \\
\hline Sill parcial & 0,075 \\
\hline No lag & 9 \\
\hline Tamaño lag & $40.000 \mathrm{~m}$ \\
\hline
\end{tabular}

(a)

\begin{tabular}{|l|r|}
\hline \multicolumn{1}{|c|}{ VALIDACIÓN CRUZADA } & \\
\hline Número de muestras & 615 \\
\hline Media de la predicción de los errores & 0,0001 \\
\hline Root Mean Square Error (RMSE) & 0,102 \\
\hline
\end{tabular}

(b)

FUENTE ELABORACIÓN PROPIA

El nugget nos indica la variabilidad aleatoria, que en este caso es cero. El valor del rango $(201.635 \mathrm{~m})$ nos pone de manifiesto que hasta esa distancia los puntos están relacionados. Además, el valor sill parcial nos indica el máximo valor promedio de la diferencia al cuadrado de los valores del factor de corrección por accesibilidad $\mathrm{u}_{1}$ entre dos puntos separados por una distancia de $201.635 \mathrm{~m}$, a partir de la cual el valor sill parcial es constante e igual a 0,075 .

\section{Spline con tensión}

En la segunda interpolación, se realizó la interpolación del factor de corrección por accesibilidad por el método Spline con tensión. Para la validez del modelo, se realizó la validación cruzada.

$\mathrm{Al}$ investigar la interpolación Spline, los parámetros principales que mejor se han ajustado al modelo se exponen en la tabla 3a) y los resultados de aplicar la validación cruzada se muestra en la tabla 3 b). 
tabla 3 | (a) Parámetros de ajuste del modelo Spline.

(b) Validación cruzada del modelo Spline

\begin{tabular}{|l|c|}
\hline \multicolumn{1}{|c|}{ PARÁMETROS } & VALORES \\
\hline Kernel & 0,0005 \\
\hline Máximos vecinos & 15 \\
\hline Mínimos vecinos & 10 \\
\hline Sector & 1 \\
\hline Eje mayor & 28000 \\
\hline Eje menor & 28000 \\
\hline Ángulo & 0 \\
\hline
\end{tabular}

(a)

FUENTE ELABORACIÓN PROPIA

\begin{tabular}{|l|c|}
\hline \multicolumn{1}{|c|}{ VALIDACIÓN CRUZADA } & \\
\hline Número de muestras & 615 \\
\hline Media de la predicción de los errores & 0,0001 \\
\hline Root mean Square Error (RMSE) & 0,118 \\
\hline
\end{tabular}

(b)

\section{Resultado de las interpolaciones Kriging Ordinario y Spline}

Al cumplir nuestros datos los requisitos de ambas interpolaciones, se procedió a realizar la interpolación del Kriging ordinario y Spline. El resultado de dichas interpolaciones se puede ver en la figura 5 . El criterio de categorización de estos mapas fue el mismo que el que se representa en la figura 2. Los valores de la variable factor de corrección por accesibilidad $\mathrm{u}_{1}$ se categorizaron en las mismas clases y con color semejante en la zonificación obtenida en ambas interpolaciones. La distribución espacial del valor del factor $\mathrm{u}_{1}$ en ambas interpolaciones es casi idéntica. La variación que se aprecia respecto al factor $\mathrm{u}_{1}$ en las dos interpolaciones es que en la interpolación Kriging ordinario el valor es superior al que resulta de la interpolación Spline, lo que afectará al valor final del suelo (figura 4).

\section{FIgURA 4 Modelo de diferencia Kriging-Spline}

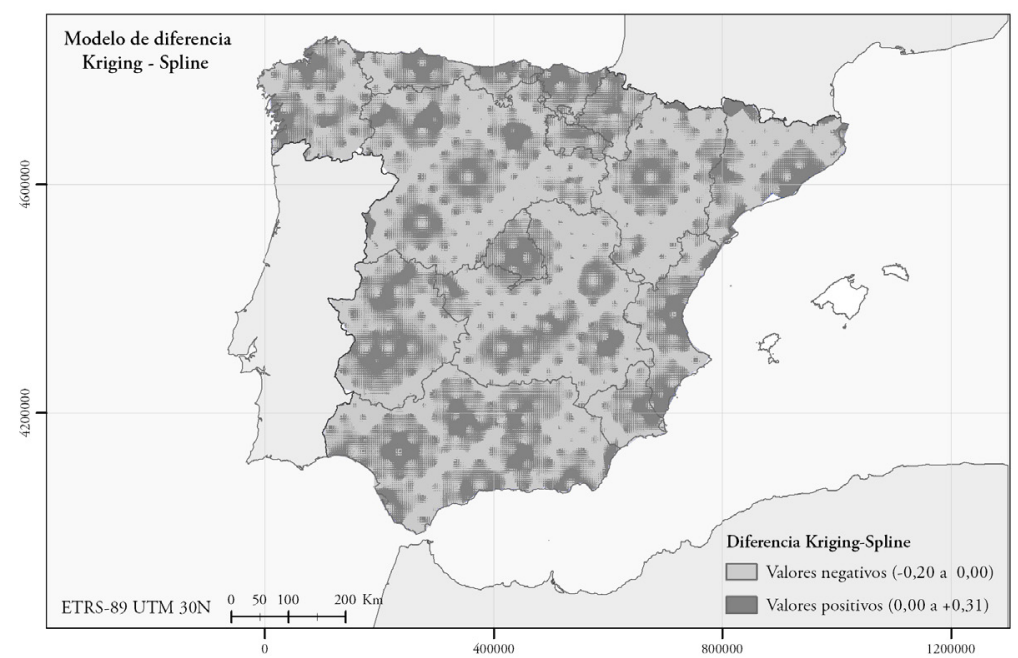

FUENTE ELABORACIÓN PROPIA 
FIGURA 5 (a) Interpolación de Kriging ordinario de la variable factor de corrección por accesibilidad $\mathbf{u}_{1}$. (b) Interpolación de Spline de la variable factor de corrección por accesibilidad $\mathbf{u}_{1}$

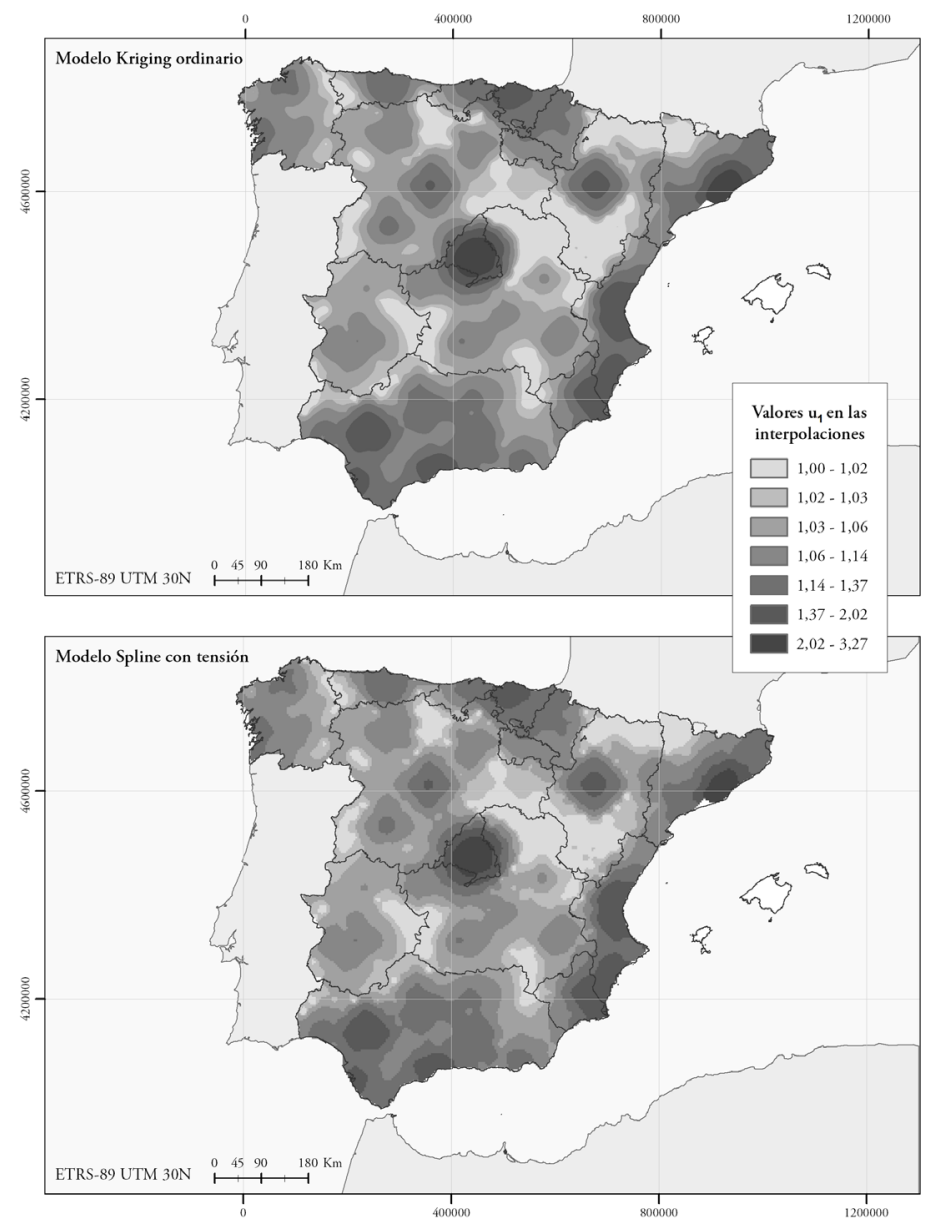

FUENTE ELABORACIÓN PROPIA

Respecto a la predicción de errores, en la comparación de los modelos pudo apreciarse cómo el método Kriging ordinario es más eficiente que el método Spline; no obstante, hay que resaltar que las diferencias son pequeñas. El error medio cuadrático (RMSE) del método Kriging ordinario es de 0,102 y el error del método Spline, 0,126.

Visualmente se aprecian las diferencias de ambos modelos mediante el diagrama de dispersión, donde el eje $\mathrm{X}$ representa los valores observados de $\mathrm{u}_{1}$ y el eje $\mathrm{Y}$, los valores estimados por sendos modelos (figura 5), observándose que el modelo Kriging ordinario (figura 6a) explica el $85 \%$ de la variabilidad de los datos. Cuando los promedios de los valores de $\mathrm{u}_{1}$ son mayores o iguales a 1,5 , la diferencia entre los valores estimados y observados genera un error negativo. Por el contrario, cuando 
la relación entre los valores estimados y observados es menor que 1,5 se generan errores positivos, deduciéndose que hay una infraestimación cuando los valores observados son altos y una sobreestimación cuando los valores son bajos.

También se puede ver cómo el modelo Spline (figura 6b), explicando el 79\% de la variabilidad de los datos, sigue una tendencia similar al modelo anterior.

FIgURA 6 | (a) Diagrama de dispersión para el modelo Kriging ordinario.

(b) Diagrama de dispersión para el modelo Spline

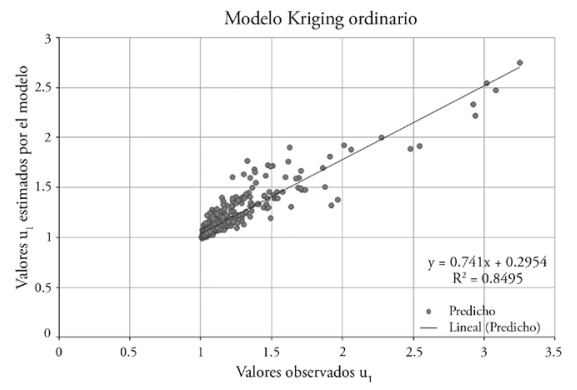

(a)

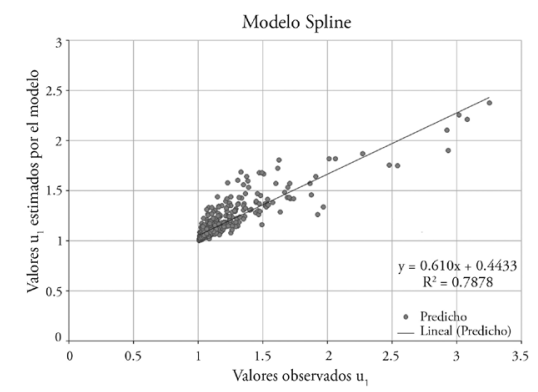

(b)

FUENTE ELABORACIÓN PROPIA

En los apartados siguientes, según lo expuesto, vamos a continuar la investigación con el modelo Kriging.

Análisis y discusión de los resultados.

\section{Estimación del factor de corrección por accesibilidad $\mathbf{u}_{1}$}

Dada la complejidad del cálculo del valor del factor de corrección por accesibilidad $\mathrm{u}_{1}$, integrante de los factores correctores que se aplican al valor de suelo calculado por la capitalización de las rentas, de acuerdo con la legislación espańola, se ha realizado un estudio del mismo en distintos puntos del territorio peninsular español, obteniendo como resultado final un mapa con el valor de este factor en dicho territorio.

Para ello se ha estudiado el valor de la variable en 615 puntos, obteniéndose valores con un rango que varía desde 1 hasta 3,27. Se ha identificado que el mayor valor de $\mathrm{u}_{1}$ se da en las ciudades de Madrid, Barcelona, Valencia, Sevilla, Málaga y Bilbao.

El impacto del transporte en el precio del territorio ha sido muy analizado. Algunos estudios (Tsutsumi \& Seya, 2008) han medido este impacto a través de herramientas de análisis espacial y datos del valor del suelo, estudiando el acceso del transporte ferroviario a las ciudades. En el presente trabajo se ha podido comprobar que existen corredores del valor $\mathrm{u}_{1}$ que se estructuran a lo largo de las autovías y autopistas. En la figura 6 se han introducido las principales carreteras, autovías y autopistas, e identificado la existencia de seis corredores en los que se recogen los valores 
más elevados del factor de corrección por accesibilidad. Estos corredores coinciden en su mayor parte con los ejes de alta velocidad ferroviaria: el corredor mediterráneo desde Murcia a la frontera catalana con Francia, el corredor Madrid-Sevilla-Cádiz coincidente con la línea de alta velocidad correspondiente, el eje Bilbao-Tarragona que se superpone al futuro corredor ferroviario Cantábrico-Mediterráneo; a ellos se ańaden los ejes León-Asturias, Vigo-La Coruña y, por último, Madrid-Bilbao, que vertebra las más importantes áreas urbanas de Castilla-León y el País Vasco.

FIGURA 7 | Ubicación de los seis corredores $\mathbf{u}_{1}$

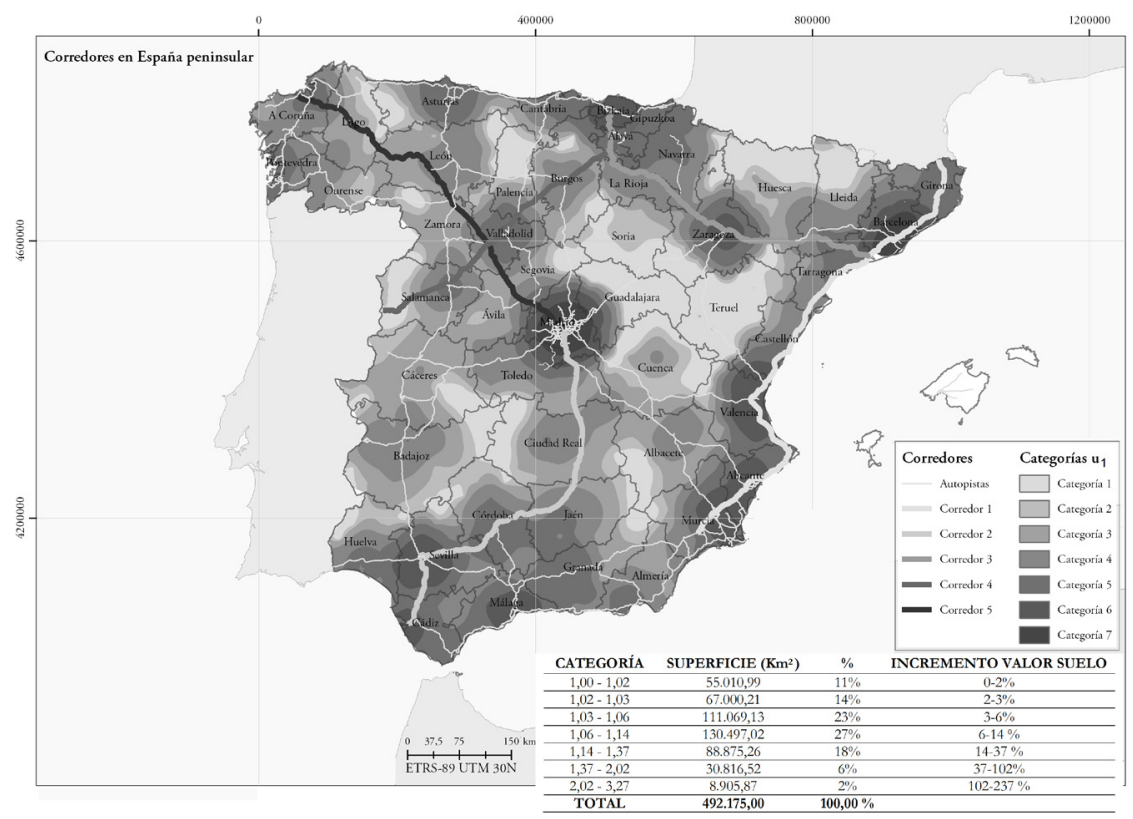

FUENTE ELABORACIÓN PROPIA

Con la interpolación realizada en este estudio se puede categorizar el territorio objeto de estudio y asignarle un incremento del valor del suelo, a efectos expropiatorios. Con la categorización de la interpolación (figura 5) se han diferenciado siete zonas según la tabla que aparece en la figura 7, en la que pueden apreciarse los incrementos de valor.

Con los resultados obtenidos en la interpolación y los corredores de carreteras, se ha encontrado una relación entre el valor $\mathrm{u}_{1} \mathrm{y}$ la densidad de carreteras (autovías, autopistas) en cada categoría. En los valores más altos del factor se presenta la mayor densidad de estas redes, y en los valores inferiores disminuye la densidad de las mismas. 


\section{Conclusiones}

Este trabajo se enmarca en el análisis del plusvalor de posición del suelo rural mediante la aplicación de un factor de corrección por accesibilidad a núcleos de población en España, según el Reglamento de valoraciones de la Ley de Suelo estatal. Para ello se han empleado técnicas de análisis espacial mediante la aplicación de un sistema de información geográfica, complementado con un análisis geoestadístico que ha permitido definir la continuidad espacial de los valores de la variable correspondiente al citado factor de corrección por accesibilidad.

La metodología aquí propuesta convierte el procedimiento discretizado previsto en la legislación (consistente en la realización de cálculos complejos con las fórmulas establecidas por la legislación española) en un modelo continuo que, mediante la interpolación, calcula el factor de localización en cualquier punto del territorio analizado. Además, el análisis geoestadístico realizado permite un análisis visual de los rangos de magnitudes resultantes del factor en relación con los elementos de atracción que activan el valor.

El mapa de interpolación Kriging (figura 5a) permite obtener el factor $\mathrm{u}_{1}$ para cualquier punto del territorio nacional. Como era previsible, este mapa pone de manifiesto que los valores mayores del factor $\mathrm{u}_{1}$ coinciden en general con los grandes núcleos de población en España, asociados a la capitalidad de las principales provincias. Obviamente, los valores máximos de la zonificación del factor $\mathrm{u}_{1}$ de los núcleos de población coinciden con las ciudades más relevantes (Madrid, Barcelona, Sevilla, Valencia, Bilbao, Málaga). Las zonas de valores mínimos del factor $\mathrm{u}_{1}$ coinciden con las zonas más despobladas de España. Con el Kriging se ha podido elegir el número de categorías o zonas más apropiadas para el estudio que se esté realizando del factor $\mathrm{u}_{1}$, como es el caso de la distribución de dicho factor por cuartiles. Además, con el método utilizado en este estudio, hemos podido cuantificar la variabilidad o autocorrelación espacial del factor $\mathrm{u}_{1} \mathrm{y}$ hemos encontrado otros factores que pueden influir en la valoración del factor $\mathrm{u}_{1}$.

El desarrollo del modelo que aquí se ha expuesto reproduce el patrón centroperiferia de la economía espacial, descrito por Fujita y Krugman (2004) como un sistema general constituido por dos tipos de ámbitos diferenciados que interactúan en un sistema de fuerzas regionales en equilibrio inestable. Por una parte, se presenta el sistema urbano, caracterizado por la actividad industrial y tecnológica propiciada por la dinámica de concentración de población y la acumulación de capitales, que opera como una fuerza centrípeta. Por otra, la periferia próxima más rururbana que periurbana- - lejana, que desarrolla una actividad agrícola de naturaleza estable, caracterizada por la dispersión de población y capitales, así como por la separación entre productores y consumidores, que opera como una fuerza centrífuga. Este modelo refleja un patrón de desigualdades territoriales, constatado en la mera distribución espacial de las rentas, y que en la norma española se refleja en dos aspectos: por una parte, en el impacto gravitacional que en el valor de suelo rural tienen las distancias a los núcleos de concentración demográfica, reflejada en el valor del factor $\mathrm{u}_{1}$; $\mathrm{y}$ por otra, en las sustanciales diferencias cuantitativas del valor económico territorial entre los dos ámbitos de referencia: el valor de la tierra 
por simple capitalización de rendimientos agrarios a largo plazo, frente la renta posicional inmediata del aprovechamiento edificatorio del suelo urbano finalista.

Los resultados obtenidos han permitido un análisis de la distribución espacial del plusvalor de posición que confirma los vínculos existentes entre los desequilibrios regionales y el nivel de desarrollo económico alcanzado, comprobándose que las fuerzas que configuran la distribución espacial de la actividad económica cuentan con una acusada inercia desde el punto de vista locacional (Fundación Bbva, 2008). Los desequilibrios poblacionales surgidos como consecuencia de los movimientos migratorios hacia los grandes centros urbanos se han reflejado en la hipertrofia de los valores territoriales, concentrados en el entorno de las capitales de las Comunidades Autónomas más populosas y económicamente activas.

Por otra parte, se ha podido comprobar también cómo los mayores valores se obtienen a lo largo de los ejes de comunicación que vertebran las áreas de mayor actividad y dinamismo económico del territorio. En este trabajo se comprueba asimismo cómo, más allá de las capacidades productivas del suelo rural, su plusvalor de posición está fuertemente condicionado por el impacto gravitacional de la distancia de los puntos de producción a los grandes centros y ejes de actividad económica de carácter urbano, y por el tamaño de estas aglomeraciones, factores que pueden incrementar hasta en un 300\% el valor resultante de los rendimientos de la producción. Estos resultados plantean, finalmente, la posibilidad de explorar nuevas hipótesis en la definición de la propuesta formulada por el Reglamento de valoraciones de la Ley del Suelo, lo que permitirá una más ajustada adecuación a los desarrollos propios de los modelos socioespaciales.

\section{Referencias bibliográficas}

Alonso, R. \& Iruretagoyena, M. T. (1995). Valoración agraria. Concepto, métodos y aplicaciones. Madrid: Mundi-Prensa.

Alonso, W. (1964). Location and land use. Toward a general theory of land rent. Cambridge, MA: Harvard University Press.

Ballesteros, E. (1991) Economía de la empresa agraria y agroalimentaria. Madrid: Mundi-Prensa. Cantillón, R. (1755) An essay on economic theory. An English translation of Richard Cantillon's Essai sur la Nature du Commerce en Général. (C. Saucier, trad.; M. Thornton, ed.). Auburn, AL: Ludwig von Mises Institute,

Caballer Mellado, V. \& Roger Fernández, G. (2012). Manual de valoraciones urbanísticas. Valencia: Tirant lo Blanch.

Caballer Mellado, V. (2008). Valoración agraria. Teoría y práctica (5 ${ }^{\mathrm{a}}$ ed.). Madrid: MundiPrensa.

Christaller, W. (1966). Central places in Southern Germany. Englewood Cliffs, nJ: Prentice Hall.

Cressie, N. (1990) The origins of kriging. Mathematical Geology, 22(3), 239-252. https://doi. org/10.1007/BF00889887

Derycke, P. (1971) L'economie urbaine. París: Presses Universitaires de France. 
Díaz Viera, M. A. (2002), Geoestadística aplicada. México, D.F.: Instituto de Geofísica, Universidad Nacional Autónoma de México (UnAm) / La Habana: Instituto de Geofísica y Astronomía; Ministerio de Ciencia, Tecnología y Medio Ambiente, Cuba.

Fujita, M. \& Krugman, P. (2004). La nueva geografía económica: pasado, presente y futuro. Investigaciones Regionales, (4). 177-206. En http://bit.ly/2pj1Tub

Fundación BBVA (2008). La distribución espacial de la actividad económica. Cuadernos Fundación BBVA. Serie Capital y Crecimiento, 9. En http://www.fbbva.es/TLFU/dat/ cd_09_08_distribucion.pdf

Ghiasi, Y. \& Nafisi, V. (2016). Strain estimation using ordinary Kriging interpolation. Survey Review, 48(350), 361-366. https://doi.org/10.1080/00396265.2015.1116155

Goodchild, M. F. \& Haining, R. P. (2004). GIS and spatial data analysis: Converging perspectives. Papers in Regional Science 83(1), 363-385. https://doi.org/10.1007/ s10110-003-0190-y

González Ruiz, R. M. (2012). Los efectos de la localización en la valoración rural: análisis de los factores de corrección. Revista de Derecho Urbanistico y Medio Ambiente, 46(273), 13-74.

Haining, R. (2003) Spatial Data Analysis. Theory and practice. Cambridge, uk. Cambridge University Press.

Harvey, D. (1977). Urbanismo y desigualdad social (Trad. M. González Arenas). Madrid: Siglo Veintiuno. En http://bit.ly/1KPOcbp

Johnston, K., Ver Hoef, J. M., Krivoruchko, K. \& Lucas, N. (2001). Using ArcGIS Geostatistical Analyst. ArcGIS 9. Redlands, CA: ESRI. En http://dusk2.geo.orst.edu/gis/geostat_ analyst.pdf

Kravchenko A. N. (2003). Influence of spatial structure on accuracy of interpolation methods. Soil Science Society of American Journal 67(5), 1564-1571. https://doi.org/10.2136/ sssaj2003.1564

Krige, D. G. (1951). A statistical approach to some basic mine valuation problems on the Witwatersrand. Journal of the Chemical, Metallurgical and Mining Society of South Africa, 52(6), 119-139. [Copia digitalizada en https://journals.co.za/content/ saimm/52/6/AJA0038223X_4792].

Lipietz, A. (1979). El capital y su espacio. México, D.F.: Siglo Veintiuno.

Lösch, A. (1954). The economics of location. New Haven, ст: Yale University Press.

Mitášová, H., \& Hofierka, J. (1993), Interpolation by regularized spline with tension: II. Application to terrain modeling and surface geometry analysis. Mathematical Geology 25(6), 657-669. https://doi.org/10.1007/BF00893172

Montero, J. M. \& Larraz, B. (2008). Introducción a la geoestadistica lineal. Perillo, La Coruña: Netbiblo, S.L.

Moral-García, F.J. (2003). La representación gráfica de las variables regionalizadas. Geoestadística lineal. Cáceres: Universidad de Extremadura.

Moreno Jiménez, A. (2007). Sistemas y Análisis de la Información Geográfica. Madrid: Ra-Ma.

Pebesma, E. J. (2006). The role of external variables and GIS databases in geostatistical analysis. Transactions in GIS 10(4), 615-632. https://doi.org/10.1111/j.14679671.2006.01015.x

Ricardo, D. (1817). The principles of political economy and taxation ( $\left.3^{\mathrm{a}} \mathrm{ed}.\right)$. Londres: John Murray

Ruiz García, F. (1986). Manual de valoración agraria y urbana. Madrid: Intervalora. 
Salazar, Z. (1950). Valoración agrícola y catastro. Madrid: Espasa-Calpe.

Santi Juárez, T. (1952). Valoración de fincas y precios. Madrid: Dossat.

Schweikert, D. G. (1966). An interpolation curve using splines in tensión. Studies in Applied Mathematics, 45(1-4), 312-317. https://doi.org/10.1002/sapm1966451312

Smith, A. (1778). An Inquiry into the Nature and Causes of the Wealth of Nations: By Adam Smith, LL. D. and frs Formerly Professor of Moral Philosoophy in the University of Glasgow; in Two Volumes (Vol. 1, The Second Edition). London: Printed from W. Strahan, and T. Cadell, in the Strand.

Tobler, W. R. (1970). A computer movie simulating urban growth in the Detroit region. Economic Geography, 46(sup1), 234-240. https://doi.org/10.2307/143141

Tsutsumi M. \& Seya, H. (2008). Measuring the impact of large-scale transportation projects on land price using spatial statistical models. Papers in Regional Science, 87(3), 385401. https://doi.org/10.1111/j.1435-5957.2008.00192.x

Weber, A. (1929). Theory of the location of industries. Chicago, IL: University of Chicago Press.

Weih, R. C. \& Dick, A. J. (2008). Modeling the pre-Euroamerican landscape with government land office surveys and geostatistics. Transactions in GIS 12(4), 443-457. https://doi. org/10.1111/j.1467-9671.2008.01115.x

\section{Legislación citada}

Real Decreto Legislativo 2/2008, de 20 de junio, por el que se aprueba el texto refundido de la Ley de suelo. En https://www.boe.es/buscar/act.php?id=BOE-A-2008-10792

Real Decreto 1492/2011, de 24 de octubre, por el que se aprueba el Reglamento de valoraciones de la Ley de Suelo. En https://www.boe.es/buscar/doc.php?id=BOE-A-2011-17629

Real Decreto Legislativo $7 / 2015$, de 30 de octubre, por el que se aprueba el texto refundido de la Ley de Suelo y Rehabilitación Urbana. En http://www.boe.es/diario_boe/txt. php?id=BOE-A-2015-11723 